\title{
Role of Vitamin C in Cardioprotection of Ischemia/Reperfusion Injury by Activation of Mitochondrial $K_{\mathrm{ATP}}$ Channel
}

\author{
Jie Hao, Wei-Wei Li, Hong Du, Zhi-Fang Zhao, Fan Liu, Jing-Chao Lu, Xiu-Chun Yang, and \\ Wei Cui* \\ The Second Hospital of Hebei Medical University; No. 215 Hepingxi Road, Shijiazhuang, Hebei Province 050000, \\ China. \\ Received September 8, 2015; accepted February 1, 2016
}

How to provide effective prevention and treatment of myocardial ischemia/reperfusion (I/R) injury and study of the mechanism underlying $I / R$ injury are hotspots of current research. This study aimed to elucidate the effect and cardioprotective mechanism of vitamin $C$ (VC) on myocardial $I / R$ injury. Our study introduced two different $I / R$ models: $I / R$ in vitro and oxygen-glucose deprivation/recovery (OGD/R) in primary neonatal rat cardiac myocytes. We used the mitochondrial permeability transition pore (mPTP) opener lonidamine (LND) and the mitochondrial $K_{\mathrm{ATP}}\left(\right.$ mitoK $\left._{\mathrm{ATP}}\right)$ channel inhibitor 5-hydroxydecanoate (5-HD) to analyze the underlying mechanisms. We found that post-treatment with VC decreased I/R injury in our models. Post-treatment with VC significantly decreased I/R-induced injury, attenuated apoptosis, and maintained the functional integrity of mitochondria via alleviation of $\mathrm{Ca}^{2+}$ overload, reactive oxygen species burst, inhibition of the opening of $\mathrm{mPTP}$, and prevention of mitochondrial membrane potential $(\Delta \Psi \mathrm{m})$ depolarization. VC post-treatment increased the phosphorylation of Akt and glycogen synthase kinase (GSK)-3 $\beta$. The present results demonstrate that $\mathrm{VC}$ might protect the myocardium from $\mathrm{I} / \mathrm{R}$-induced injury by inhibiting the $\mathrm{mPTP}$ opening via activation of mitoK $\mathrm{K}_{\mathrm{ATP}}$ channels. $\mathrm{VC}$ mediates cardioprotection via activation of the phosphatidyl inositol 3-kinase (PI3K)-Akt signaling pathway. These findings may contribute toward the development of novel strategies for clinical cardioprotection against $\mathbf{I} / \mathbf{R}$ injury.

Key words vitamin $\mathrm{C}(\mathrm{VC})$; ischemia/reperfusion (I/R) injury; ischemic post-treatment; mitochondrial $\mathrm{K}_{\mathrm{ATP}}$ $\left(\right.$ mitoK $\left._{\text {ATP }}\right)$ channel

In recent years, cardiovascular disease has become a great threat to human life and health. Prolonged myocardial ischemia can cause tissue damage and even cell death. A key treatment of coronary heart disease is blood reperfusion of the myocardium. However, restoration of blood supply followed by myocardial cell function and structural damage results in a pathophysiological state known as ischemia/reperfusion (I/R) injury. ${ }^{1)}$ The reduction occurrence of I/R injury has become a focus of current research.

In 1986, Murry first put forward a self-defensive protective mechanism of the myocardial cell known as myocardial ischemia preadaptation (ischemic preconditioning; IPC), and transient ischemic preconditioning can improve myocardial ischemia and reperfusion of longer than damage tolerance ability. ${ }^{2)}$ However, ischemic preconditioning poses challenges including safety and operability, and individual differences of clinical application of IPC is restricted. Thus, several studies have proposed drug post-treatment (pharmacological preconditioning; PPC), namely the simulation of IPC via a variety of drugs result in less I/R injury. ${ }^{3)}$

In myocardial $\mathrm{I} / \mathrm{R}$ injury, the integrity of the mitochondrial membrane and its functions have undergone major changes, such as depolarization of the mitochondrial membrane potential $(\Delta \Psi \mathrm{m})$, interruption of mitochondrial respiratory chain electron transfer, inhibition of ATP synthesis and the release of pro-apoptosis proteins. ${ }^{3)}$ Many studies have confirmed the mitochondria as an important target of heart protection. ${ }^{4,5}$ The irreversible damage of mitochondria results in cell death. Protection of mitochondrial structure and function contributes to a reduced degree of damage after I/R. Several studies have shown that mitochondrial ATP-sensitive potassium channels (mitoK $\mathrm{K}_{\mathrm{ATP}}$ channels) and the mitochondrial permeability transition pore (mPTP) are involved in the cardioprotection of IPC. ${ }^{6-8)}$

Although the mechanism of IPC is still not entirely clear, $\mathrm{K}_{\text {ATP }}$ channels are known targets of IPC. After IPC and reperfusion, $\mathrm{K}_{\mathrm{ATP}}$ channels of the myocardial cell remain open, and the $\mathrm{K}_{\mathrm{ATP}}$ channels blocker benzene urea can inhibit IPC myocardial protection. This result suggested that the opening of $\mathrm{K}_{\text {ATP }}$ channels play an important role in IPC. ${ }^{9)}$ Moreover, a study found that during the ischemic period, MPTP is closed, but in the early stage of reperfusion, MPTP is in the open state. ${ }^{10)}$ Several studies have shown that mPTP plays an important role in myocardial $\mathrm{I} / \mathrm{R}$ injury. High $\mathrm{pH}$ value, depletion of ATP, high levels of phosphate, calcium overload, and an outbreak of reactive oxygen species (ROS) can induce mPTP to open. Calcium overload and ROS are the main inducing factors. ${ }^{11)}$

In this study, we observed the effects of vitamin C (VC) in two levels of rat $\mathrm{I} / \mathrm{R}$ models: $\mathrm{I} / \mathrm{R}$ in vitro and oxygen-glucose deprivation/recovery (OGD/R) in primary neonatal rat cardiac myocytes and we investigated the mechanisms of VC underlying the cardioprotective effects using confocal laser-scanning microscopy, flow cytometry and biochemical and morphological methods. We found that VC post-treatment after ischemia had a similar cardioprotective effect to IPC in I/R model in vivo. Our study also showed that post-treatment of $\mathrm{VC}$ may have a cardioprotective effect via mimicking the protective mechanisms of IPC. 


\section{Experimental}

The use of animals was approved by Hebei Province Experimental Animal Management Methods and the Laboratory Animal Centre of Hebei Province.

\section{Studies in Isolated Perfused Heart}

$\mathrm{I} / \mathrm{R}$ in Isolated Heart

Male Sprague-Dawley (SD) rats $(250-300 \mathrm{~g})$ were anesthetized with sodium pentobarbital $(50 \mathrm{mg} / \mathrm{kg}$, intraperitoneally (i.p.)). An aortic cannula was inserted into the aorta, with fixed line 4 ligation, to make the heart hang from the Langendorff device, at a rate of $8 \mathrm{~mL} / \mathrm{min}$ in a circular manner with constant speed retrograde perfusion. The Langendorff contained Krebs-Henseleit $(\mathrm{K}-\mathrm{H})$ solution, which consists of: $\mathrm{NaCl} 118 \mathrm{~mm}, \mathrm{KCl} 4.7 \mathrm{~mm}, \mathrm{MgSO}_{4} 1.2 \mathrm{~mm}, \mathrm{KH}_{2} \mathrm{PO}_{4} 1.2 \mathrm{~mm}$, $\mathrm{CaCl}_{2} 1.8 \mathrm{~mm}, \mathrm{NaHCO}_{3} 25 \mathrm{~mm}$ and glucose $10 \mathrm{~mm}$. The $\mathrm{K}-\mathrm{H}$ buffer was continuously gassed with $95 \% \mathrm{O}_{2}$ and $5 \% \mathrm{CO}_{2}$ at $37^{\circ} \mathrm{C}$. After re-beating of the heart, a latex balloon was introduced into the left ventricle via the left atrium and mitral valve. The balloon volume was connected to a pressure transducer, resulting in a left ventricular end-diastolic pressure (LVEDP) between 4 and $12 \mathrm{mmHg}$. The balloon capacity was constant during the experimental process. All hearts were stabilized for at least $15 \mathrm{~min}$. The left ventricular systolic pressure (LVSP), LVEDP, the maximal differentials of LVSP $( \pm \mathrm{d} p /$ $\left.\mathrm{d} t_{\text {max }}\right)$ and heart rate (HR) were monitored using the PowerLab system (AD Instruments Ltd., Australia).

After the reperfusion period, the activity of lactate dehydrogenase $(\mathrm{LDH})$ in the coronary effluent was detected using a commercial kit (Jiancheng, China). The infarct area (IA) was determined using triphenyl-tetrazolium chloride (TTC). Oxidized form of nicotinamide adenine dinucleotide ${ }^{+}\left(\mathrm{NAD}^{+}\right)$ content, a marker of mPTP opening, was measured using the $\mathrm{NAD}^{+}$Assay Kit (Biochain). The terminal deoxynucleotidyl transferase-mediated deoxyuridine triphosphate nick-end labeling (TUNEL) apoptosis assay kit (Beyotime, China) was used to detect the apoptosis level in the myocardium according to the manufacturer's instructions.

Experimental Protocol

Post-experiments have shown that VC $(2 \mu \mathrm{M})$, added to $\mathrm{K}-\mathrm{H}$ buffer for $30 \mathrm{~min}$ after global ischemia, exerts the optimal cardioprotective effect. Male SD rats $(250-300 \mathrm{~g})$ were randomly divided into 7 groups $(n=5)$ :

(1) Control group: isolated hearts were perfused without $\mathrm{I} / \mathrm{R}$;

(2) I/R group: hearts underwent $30 \mathrm{~min}$ global ischemia followed by $120 \mathrm{~min}$ of reperfusion;

(3) VC group: hearts were post-treated with $2 \mu \mathrm{M} \mathrm{VC}$ for $30 \mathrm{~min}$ after global ischemia;

(4) VC+5-hydroxydecanoate (5-HD) group: hearts were perfused for $20 \mathrm{~min}$ with $100 \mu \mathrm{m}$ 5-HD before global ischemia and then treated with $2 \mu \mathrm{M}$ VC for $30 \mathrm{~min}$ before reperfusion;

(5) $\mathrm{VC}+$ lonidamine (LND) group: hearts were treated with $2 \mu \mathrm{M}$ VC for $30 \mathrm{~min}$ after global ischemia and exposed to $30 \mu \mathrm{M}$ LND for $20 \mathrm{~min}$ at the beginning of reperfusion;

(6) 5-HD group: hearts were first perfused for $20 \mathrm{~min}$ with $100 \mu \mathrm{M} 5$-HD and then perfused with $\mathrm{K}-\mathrm{H}$ buffer for $30 \mathrm{~min}$ before global ischemia;

(7) LND group: hearts were exposed to $30 \mu \mathrm{M}$ LND for $20 \mathrm{~min}$ at the beginning of reperfusion.

In this protocol, 5-HD or LND were added to the $\mathrm{K}-\mathrm{H}$ buffer, has been shown in previous studies to inhibit mitoK $\mathrm{ATP}_{\mathrm{AT}}$ channels $^{12)}$ or open mPTP. ${ }^{13)}$

Myocardial Mitochondrial Oxidative Phosphorylation Function Test

Following the above protocol, myocardial mitochondria were isolated and its oxygen consumption was evaluated in state 3 (phosphorylating state) and state 4 (resting state) in order to calculate the respiratory control index (RCI). Myocardial mitochondria were isolated by differential centrifugation. Respiratory measurements were carried out in $3.5 \mathrm{~mL}$ of air-saturated medium with $250 \mathrm{~mm}$ sucrose, $150 \mathrm{~mm}$ sodium succinate, $2 \mathrm{~mm} \mathrm{MgCl}_{2}, 0.1 \mathrm{M} \mathrm{KH}_{2} \mathrm{PO}_{4}, 3.4 \mathrm{~mm}$ Tris- $\mathrm{HCl}, 1 \mathrm{~mm}$ $\mathrm{ADP}$, and mitochondria suspension. Oxygen consumption was determined using a Clark-type oxygen electrode. Data are expressed as the RCI, which is a relative value of state 3 and state 4 that indicates the respiratory coupling in availability of ADP.

\section{Studies in the OGD/R Cell Model}

Primary Culture of Neonatal Rat Cardiac Ventricular Myocytes

Cardiac ventricular myocytes were prepared from 1 to $3 \mathrm{~d}$-old neonatal SD rats as previously described.,14)

$\mathrm{OGD} / \mathrm{R}$

$\mathrm{OGD} / \mathrm{R}$ was performed on neonatal rat ventricular myocytes using previously described methods ${ }^{15,16)}$ with modifications. The cell medium was replaced with glucose-free Dulbecco's modified Eagle's medium (DMEM), which was pre-conducted with a mixture of $5 \% \mathrm{CO}_{2}$ and $95 \% \mathrm{~N}_{2}$ for $20 \mathrm{~min}$ to remove $\mathrm{O}_{2}$. Next, cells were cultured in an incubator containing $5 \% \mathrm{CO}_{2}$ and $95 \% \mathrm{~N}_{2}$, maintained at $37^{\circ} \mathrm{C}$ for $8 \mathrm{~h}$. Next, the medium was replaced with DMEM-containing glucose, and cells were transferred into an incubator with $5 \%$ $\mathrm{CO}_{2}$ at $37^{\circ} \mathrm{C}$ for $3 \mathrm{~h}$.

Experimental Protocol

Previous experiments have shown that the addition of VC $(2 \mu \mathrm{M})$ to the culture medium after OGD for $3 \mathrm{~h}$ exerts the optimal cardioprotective effect. The experiment was divided into 7 groups:

(1) Control group: cells were cultured under normal conditions;

(2) OGD/R group: cells were subjected to $8 \mathrm{~h}$ OGD and $3 \mathrm{~h}$ recovery only;

(3) VC group: cells were post-conditioned with normal culture medium containing $2 \mu \mathrm{M}$ VC for $30 \mathrm{~min}$ after OGD; and followed by $2.5 \mathrm{~h}$ reperfusion;

(4) VC+5-HD group: the cells were first treated with $100 \mu \mathrm{M} 5$-HD for $20 \mathrm{~min}$, before OGD; at the beginning of reperfusion, the cells were incubated for $30 \mathrm{~min}$ with $2 \mu \mathrm{M} \mathrm{VC}$;

(5) VC+LND groups: after undergoing OGD for $8 \mathrm{~h}$, the cells were grown with normal culture medium. Cells were incubated with $2 \mu \mathrm{M} \mathrm{VC}$ and $30 \mu \mathrm{M}$ LND for $30 \mathrm{~min}$ and followed by $2.5 \mathrm{~h}$ reperfusion;

(6) 5-HD group: before OGD, the cells were incubated with $100 \mu \mathrm{M} 5$-HD for $20 \mathrm{~min}$; the medium was then replaced twice with DMEM medium. Next, the cells were perfused for $3 \mathrm{~h}$;

(7) LND group: cells were exposed to OGD for $8 \mathrm{~h}$, followed by incubation with $30 \mu \mathrm{M}$ LND for $30 \mathrm{~min}$. Next, the cells were perfused for $2.5 \mathrm{~h}$.

Cell Viability Assay

Following the above cell treatment protocol, cells were assessed for viability using the 3-(4,5-dimethylthiazol-2-yl)-2,5- 

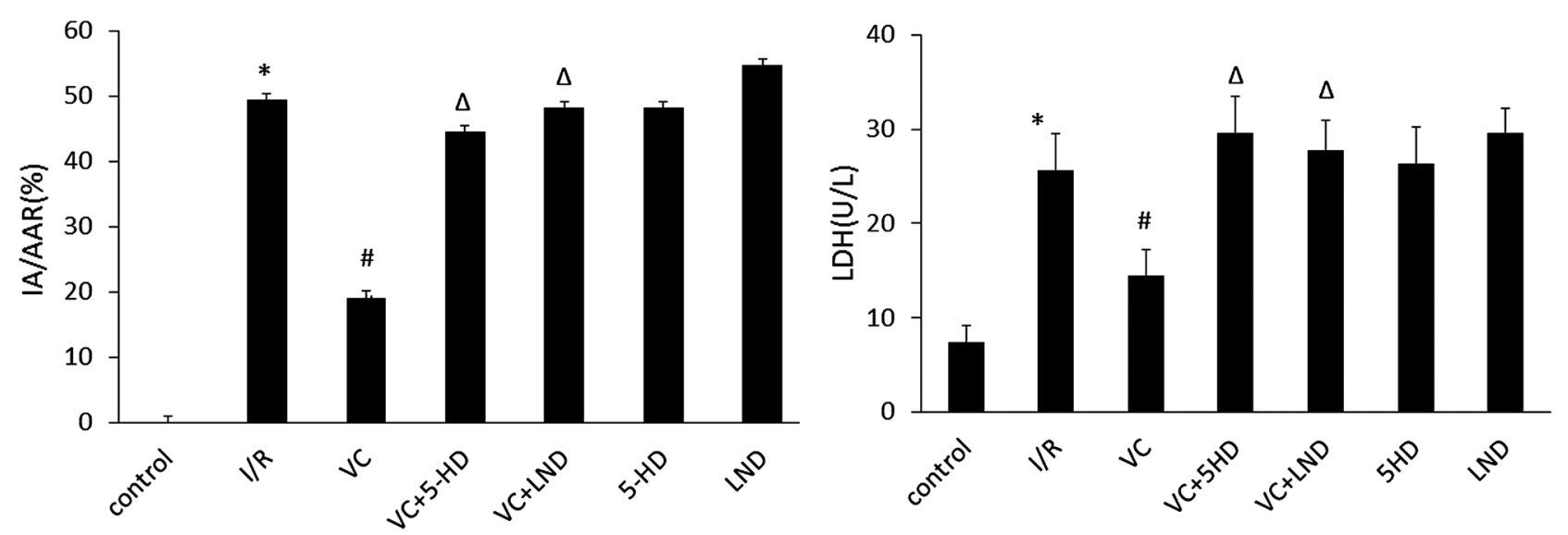

Fig. 1. Cardioprotective Effects of VC after I/R in Vitro

(A) Effects of VC on infarct area (IA) as a percentage of area at risk (AAR). (B) Effects of VC on LDH activity in isolated rat hearts subjected to I/R in vitro. Data are expressed as the mean \pm S.D., $n=5$. ${ }^{*} p<0.05$ vs. control group; ${ }^{\#} p<0.05$ vs. I/R group; ${ }^{\Delta} p<0.05$ vs. VC group. I/R: ischemia/reperfusion; VC: vitamin C; 5 -HD: 5 -hydroxydecanoic acid; LND: lonidamine.

diphenyltetrazolium bromide (MTT) reagent. Cells were cultured for $4 \mathrm{~h}$. Next, the medium was removed and dimethyl sulfoxide (DMSO) $(150 \mu \mathrm{L})$ was added into each well. Formazan concentration, which is directly proportional to cell viability, was measured at $492 \mathrm{~nm}$.

\section{LDH Activity Assay}

The supernatant was acquired from the culture medium by centrifugation for $1 \mathrm{~min}$ after recovery for $3 \mathrm{~h}$. A kit was used to detect the activity of LDH in the supernatant

$\mathrm{Ca}^{2+}$ Concentration Assay

The $\mathrm{Ca}^{2+}$ concentration was measured using a $\mathrm{Ca}^{2+}$-sensitive fluorescent probe, as previously described. ${ }^{17,18)}$ To measure the cytosolic $\mathrm{Ca}^{2+}$ concentration, the cells were incubated with $20 \mu \mathrm{M}$ Fluo-4/AM and $1 \mu \mathrm{M}$ pluronic F-127 for $20 \mathrm{~min}$ at $37^{\circ} \mathrm{C}$ in the dark. Fluorescence images were obtained using confocal laser-scanning microscopy, with $488 \mathrm{~nm}$ excitation wavelength and $510 \mathrm{~nm}$ detection wavelength.

To measure the mitochondrial $\mathrm{Ca}^{2+}$ concentration, the cells were incubated with $5 \mu \mathrm{M}$ Rhod-2/AM and $1 \mu \mathrm{M}$ pluronic F-127 for $20 \mathrm{~min}$ at $37^{\circ} \mathrm{C}$ in the dark. Fluorescence images were obtained using confocal laser-scanning microscopy, with $543 \mathrm{~nm}$ excitation wavelength and $570 \mathrm{~nm}$ detection wavelength.

ROS Concentration Assay

The intracellular reactive oxygen species level was measured using a carboxy $2^{\prime}, 7^{\prime}$-dichlorodihydrofluorescein diacetate (H2DCFDA) probe, as previously described. ${ }^{18)}$ The cells were cultured with $10 \mu \mathrm{M}$ carboxyH2DCFDA for $20 \mathrm{~min}$ at $37^{\circ} \mathrm{C}$ in the dark. CarboxyH2DCFDA is non-fluorescent and ROS can oxidize it to dichloro fluorescein (DCF), which emits a green fluorescence. The fluorescent signal intensity of DCF was detected using a flow cytometer.

Mitochondrial Permeability Transition Pore (mPTP) Opening Assay

We used a previously established method to detect the mPTP opening in an intact cell. ${ }^{19)}$ After recovery for $20 \mathrm{~min}$, the cells were incubated with $1.0 \mu \mathrm{M}$ calcein/AM and $2 \mathrm{mM}$ $\mathrm{CoCl}_{2}$ for $20 \mathrm{~min}$ in the dark. When the mPTP opened, Calcein in the mitochondria entered into the cytoplasm via the mPTP and was quenched by $\mathrm{CoCl}_{2}$ in the cytoplasm. Using confocal laser scanning microscopy, we tested the fluores- cence intensity, which reflected the extent of mPTP opening. The change in fluorescence intensity is inversely proportional with extent of opening of the MPTP. Images were analyzed using a laser-scanning confocal microscope (Nikon) with a $\times 63 / 1.4$ numerical aperture Apo oil objective lens. Also, fluorescent intensity of the region of interest was acquired using LAS AF software (Leica).

\section{$\Delta \Psi \mathrm{m}$ Assay}

$\Delta \Psi \mathrm{m}$ was detected by Tetramethylrhodamine ethylester (TMRE), which distributes across the inner mitochondrial membrane. The cells were incubated with $100 \mathrm{nM}$ TMRE at $37^{\circ} \mathrm{C}$ for $20 \mathrm{~min}$ in the dark. Fluorescence intensity was recorded using a helium/neon $(\mathrm{He} / \mathrm{Ne})$ laser, with an excitation at 543 and $560 \mathrm{~nm}$ detection wavelength. Thirty cells were randomly selected in each experiment by drawing regions around individual cells. All results were normalised and expressed as a percentage of the average fluorescence intensity reading from the control group.

Western Blotting Analyses

The experimental group: (1) Control group: cells were cultured under normal conditions; (2) OGD/R group: cells were subjected to $8 \mathrm{~h}$ OGD and $3 \mathrm{~h}$ recovery only; (3) VC group: cells were post-conditioned with $2 \mu \mathrm{M}$ VC for $30 \mathrm{~min}$ after OGD; and followed by a $2.5 \mathrm{~h}$ reperfusion; (4) VC+LY294002 groups: after undergoing OGD for $8 \mathrm{~h}$, cells were cultured with normal culture medium containing $2 \mu \mathrm{M} \mathrm{VC}$ and $15 \mu \mathrm{M}$ LY294002 for $30 \mathrm{~min}$, followed by a $2.5 \mathrm{~h}$ reperfusion; (5) LY294002 group: cells were exposed to OGD for $8 \mathrm{~h}$, followed by incubated with $15 \mu \mathrm{M}$ LY294002 for $30 \mathrm{~min}$. Next, the cells underwent reperfusion for $2.5 \mathrm{~h}$; (6) $\mathrm{VC}+5-\mathrm{HD}$ group: the cells were first treated with $100 \mu \mathrm{M} 5$-HD for $20 \mathrm{~min}$, before OGD; at the beginning of reperfusion, the cells were incubated for $30 \mathrm{~min}$ with $2 \mu \mathrm{M} \mathrm{VC}$; (7) 5-HD group: before OGD, the cells were incubated with $100 \mu \mathrm{M} 5$-HD for $20 \mathrm{~min}$; the medium was then replaced twice with DMEM medium. Next, the cells were perfused for $3 \mathrm{~h}$.

Cells were lysed at $4^{\circ} \mathrm{C}$ with radio immunoprecipitation assay (RIPA) lysis buffer containing $1 \mu \mathrm{M}$ phenylmethylsulfonyl fluoride (PMSF). Equal amounts of protein were separated using sodium dodecyl sulfate-polyacrylamide gel electropho- 
A

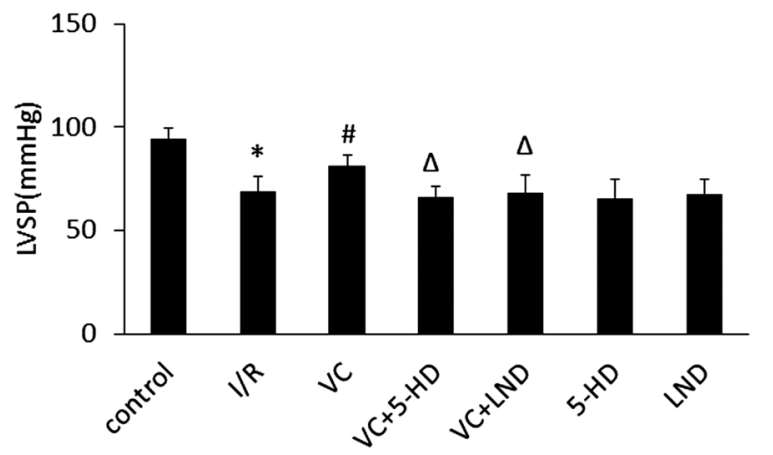

C

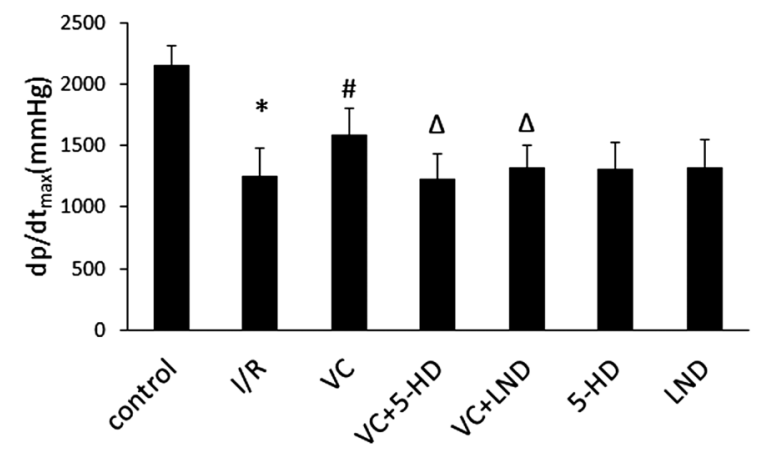

E

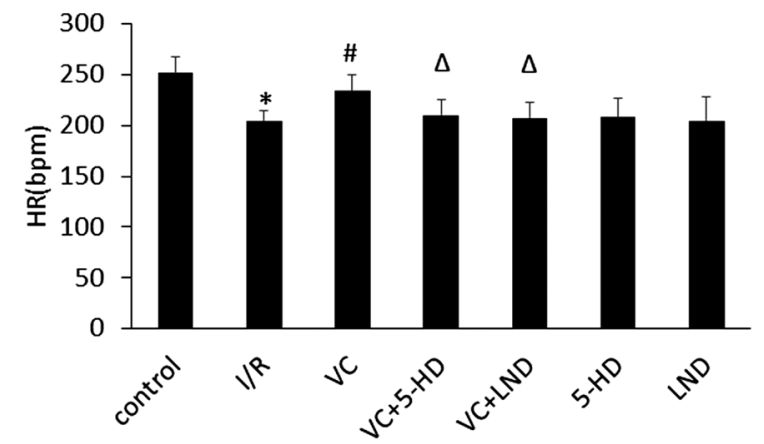

B

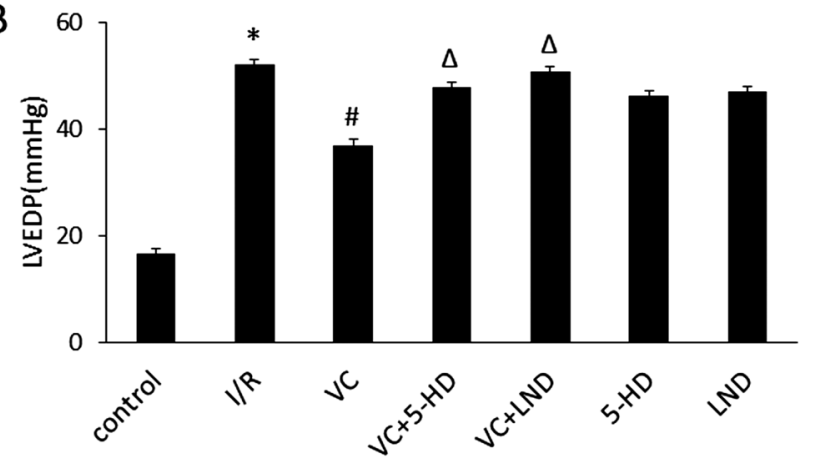

D

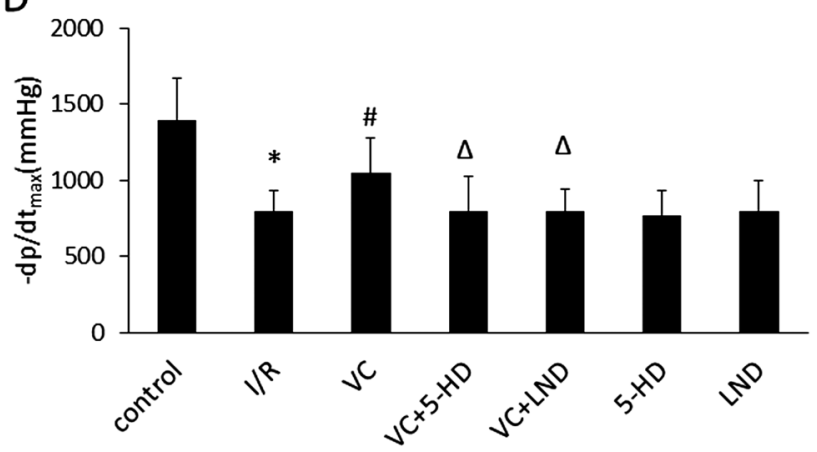

Fig. 2. Effects of VC on Hemodynamic Parameters

Data are expressed as the mean \pm S.D., $n=5$. ${ }^{*} p<0.05$ vs. control group; ${ }^{*} p<0.05 v s$. I/R group; ${ }^{\Delta} p<0.05$ vs. VC group. I/R: ischemia/reperfusion; VC: Vitamin C; 5-HD: 5-hydroxydecanoic acid; LND: lonidamine; LVSP: left ventricular systolic pressure; LVEDP: left ventricular enddiastolic pressure; $\mathrm{d} p / \mathrm{d} t_{\text {max }}$ : maximal increase rate of LVSP; $-\mathrm{d} p / \mathrm{d} t_{\min }$ : maximal decrease rate of LVSP, HR: heart rate.

resis (SDS-PAGE) and electrotransferred onto a polyvinylidene fluoride (PVDF) membrane. The membrane was sealed with Tris buffered saline (TBS) containing 5\% skimmed milk powder for $1 \mathrm{~h}$ and incubated with primary antibodies: pAkt $\left(\mathrm{Ser}^{473}\right)$ or phospho-glycogen synthase kinase (pGSK)-3 $\beta$ $\left(\mathrm{Ser}^{9}\right)$ at $4^{\circ} \mathrm{C}$ overnight. The membrane was washed with TBST (TBS, pH 7.5, containing 0.1\% Tween-20) and incubated with a secondary antibody for $1 \mathrm{~h}$. Immobilon western chemiluminescent horseradish peroxidase (HRP) substrate was used to detect peroxidase activity.

Statistical Analysis Data were expressed as the mean \pm standard deviation (S.D.) All data were analyzed using one-way ANOVA followed by Bonferroni post-hoc test (SPSS software package version 17.0). Statistical significance was considered at $p<0.05$.

\section{Results and Discussion \\ In Vitro Experiments}

Effects of VC on I/R-Induced Infarct Area (IA) and LDH Activity in Isolated Rat Hearts

Compared with the I/R group, both the IA as a percentage of area at risk (AAR) and LDH activity in the VC group were all significantly reduced $(p<0.05)$. The mitoK ${ }_{\mathrm{ATP}}$ channel inhibitor 5-HD or the mPTP opener LND significantly abolished the effects of $\mathrm{VC}(p<0.05)$. However, 5-HD or LND alone had no apparent effect on the infarct area and activity of LDH $(p<0.05$, Figs. 1A, B).

Effects of VC on Hemodynamics

Baseline hemodynamics among all experimental groups were not significantly different $(p>0.05)$. Compared with the control group, the values of LVSP, HR, $\mathrm{d} p / \mathrm{d} t_{\max }$ and $-\mathrm{d} p / \mathrm{d} t_{\max }$ decreased, whereas LVEDP increased significantly in $I / R$ groups (Fig. 2). Compared with $\mathrm{I} / \mathrm{R}$ group, all hemodynamic variables were better in the VC group $(p<0.05)$. VC-induced protection of cardiac function was completely abolished by either 5-HD or LND (Fig. 2). 
Effects of $\mathrm{VC}$ on the $\mathrm{NAD}^{+}$Content in Myocardial Tissue

The $\mathrm{NAD}^{+}$content is a marker of mPTP opening. As shown in Fig. 3, the content of $\mathrm{NAD}^{+}$in $\mathrm{I} / \mathrm{R}$ group and all of the drug treatment group were lower compared to the control group $(p<0.05)$. The content of $\mathrm{NAD}^{+}$in the $\mathrm{VC}$ group was significantly increased $(p<0.05)$ compared with I/R group, which suggested that VC inhibited mPTP opening. Furthermore, 5-HD or LND abolished the inhibitory effect of VC on mPTP opening. 5-HD or LND had no effect on mPTP opening when used alone $(p>0.05)$.

Effects of VC on Apoptosis in the Myocardium

We next examined whether VC had any protective effects on the apoptosis levels of the myocardium using TUNEL staining. The number of TUNEL-positive cells in I/R group was markedly increased compared with the control group $(p<0.05)$, whereas VC statistically decreased the number of

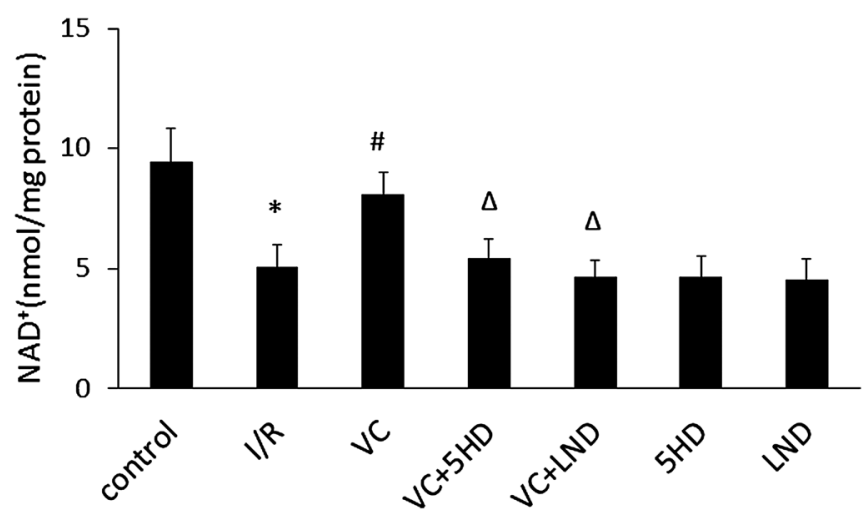

Fig. 3. Effects of $\mathrm{VC}$ on $\mathrm{NAD}^{+}$Content in Hearts Subjected to I/R

Data are expressed as the mean \pm S.D., $n=5$. ${ }^{*} p<0.05$ vs. control group; ${ }^{*} p<0.05$ vs. I/R group; ${ }^{\Delta} p<0.05$ vs. VC group. I/R: ischemia/reperfusion; VC: vitamin C; 5-HD: 5-hydroxydecanoic acid; LND: lonidamine. myocardium TUNEL-positive cells $(p<0.05)$ (Fig. 4). 5-HD or LND abolished the cardioprotection of VC. 5-HD or LND had no effect on cardioprotection when used alone.

Effect of VC on Myocardial Mitochondrial Oxidative Phosphorylation Function

The RCI was lessened in I/R group $(1.83 \pm 0.23)$ in comparison with control group $(2.51 \pm 0.06)$. The post-treatment of $\mathrm{VC}$ induced a significant increase in oxygen consumption $(2.22 \pm 0.13)$ in comparison to $\mathrm{I} / \mathrm{R}$ group $(1.83 \pm 0.23)$. However, $\mathrm{VC}+5-\mathrm{HD}$ group and $\mathrm{VC}+\mathrm{LND}$ group showed a lower RCR $(1.67 \pm 0.15$ and $1.87 \pm 0.10)$ in comparison to VC group (2.22 \pm 0.13$)$. 5-HD group and LND group showed no significant difference in RC $(1.64 \pm 0.17$ and $2.01 \pm 0.14)$ in comparison with I/R group (Table 1).

\section{Studies in the OGD/R Cell Model}

Effects of VC on OGD/R-Induced Cell Injury

As shown in Fig. 5A, treatment with $2 \mu \mathrm{M}$ VC for $3 \mathrm{~h}$ after OGD significantly increased the cell viability as assessed using the MTT assay. 5-HD or LND significantly inhibited the

Table 1. Effect of VC on the Myocardial Mitochondrial Respiratory Control Index

\begin{tabular}{lcll}
\hline \hline & S3 & \multicolumn{1}{c}{ S4 } & RCI \\
\hline C & $4.13 \pm 0.07$ & $1.65 \pm 0.16$ & $2.51 \pm 0.06$ \\
I/R & $2.72 \pm 0.11^{*}$ & $1.49 \pm 0.29^{*}$ & $1.83 \pm 0.23^{*}$ \\
VC & $4.35 \pm 0.23^{\#}$ & $1.96 \pm 0.17^{\#}$ & $2.22 \pm 0.13^{\#}$ \\
VC+5-HD & $2.31 \pm 0.24^{\triangle}$ & $1.38 \pm 0.23^{\triangle}$ & $1.67 \pm 0.15^{\triangle}$ \\
VC+LND & $3.01 \pm 0.17^{\triangle}$ & $1.61 \pm 0.30^{\triangle}$ & $1.87 \pm 0.10^{\triangle}$ \\
5-HD & $2.59 \pm 0.32^{*}$ & $1.58 \pm 0.09^{*}$ & $1.64 \pm 0.17^{*}$ \\
LND & $2.87 \pm 0.04^{*}$ & $1.43 \pm 0.12^{*}$ & $2.01 \pm 1.04^{*}$ \\
\hline
\end{tabular}

Data are expressed as the mean \pm S.D., $n=5 .{ }^{*} p<0.05 v s . \mathrm{I} / \mathrm{R}$ group; ${ }^{\#} p<0.05 v s$. VC group. I/R: ischemia/reperfusion; VC: vitamin C; 5-HD: 5-hydroxydecanoic acid; LND: lonidamine.

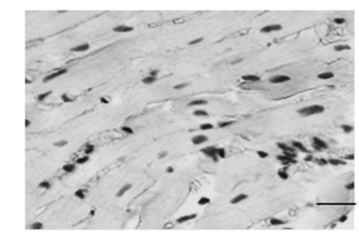

control

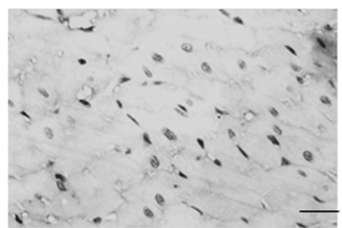

$\mathrm{VC}+5-\mathrm{HD}$

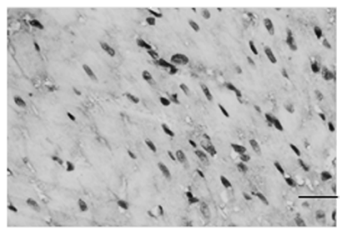

5-HD

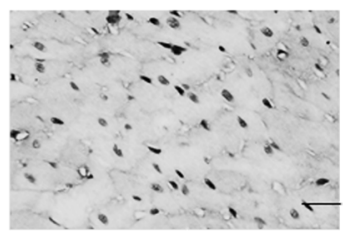

$\mathrm{I} / \mathrm{R}$

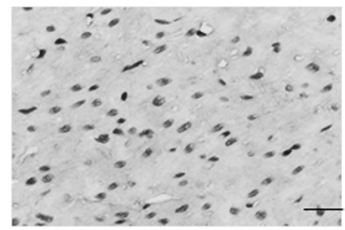

VC+LND

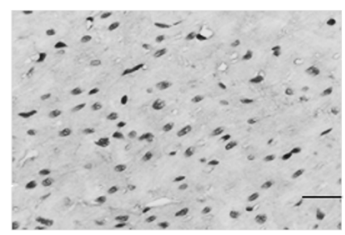

LND

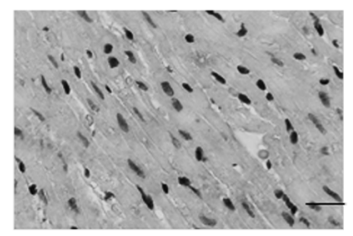

VC

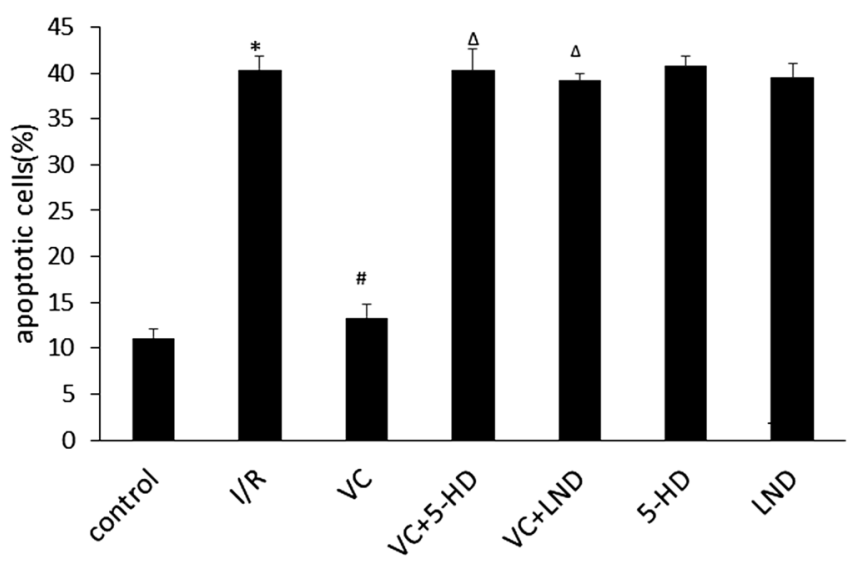

Fig. 4. Effects of VC on Apoptosis in the Myocardium

Data are expressed as the mean \pm S.D., $n=5$. ${ }^{*} p<0.05 v s$. control group; ${ }^{*} p<0.05 v s$. I/R group; ${ }^{\Delta} p<0.05$ vs. VC group. I/R: ischemia/reperfusion; VC: vitamin C; 5 -HD: 5-hydroxydecanoic acid; LND: lonidamine. Bar length, $50 \mu \mathrm{m}$. 


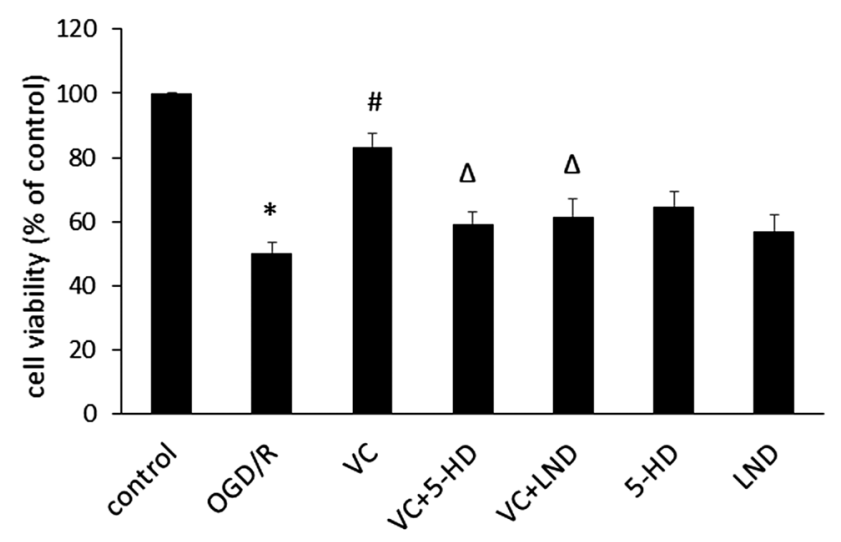

B

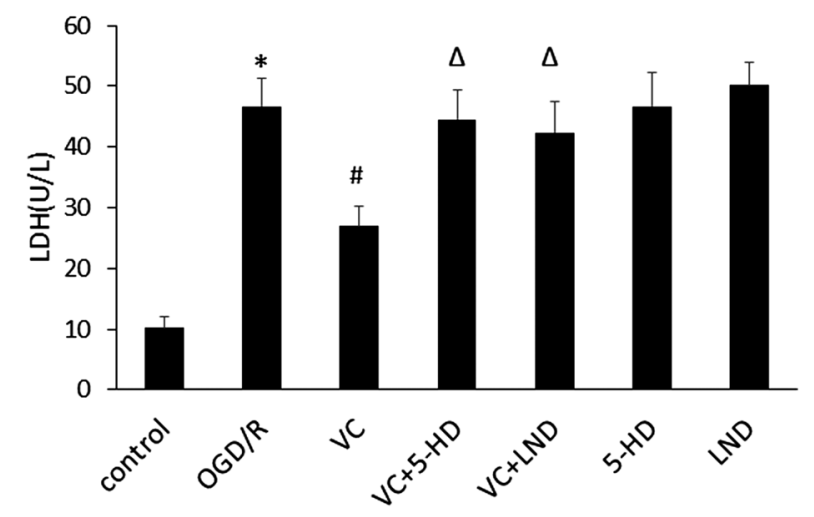

Fig. 5. Effects of VC on OGD/R-Induced Cell Injury

(A) Cell viability assessed using the MTT assay. (B) Effects of VC on lactate dehydrogenase (LDH) activity in primary cardiomyocytes after OGD/R damage. Data are expressed as the mean \pm S.D., $n=5$. ${ }^{*} p<0.05$ vs. control group; ${ }^{\#} p<0.05$ vs. I/R group; ${ }^{\Delta} p<0.05$ v. VC group. I/R: ischemia/reperfusion; VC: vitamin C; 5 -HD: 5 -hydroxydecanoic acid; LND: lonidamine.

protection of VC on cardiomyocytes (Fig. 5A). 5-HD or LND had no apparent effect on cell viability when used alone.

The activity of LDH in culture medium could also be used as a marker of cell injury. As shown in Fig. 5B, LDH activity was significantly increased in the $\mathrm{OGD} / \mathrm{R}$ group compared with the control group $(p<0.05)$. The VC group reduced LDH activity, and its effect was completely abolished by $5-\mathrm{HD}$ or LND.

Effects of VC on OGD/R-Induced $\mathrm{Ca}^{2+}$ Overload

It is well known that $\mathrm{Ca}^{2+}$ is an important regulator of mPTP opening. Thus, we investigated the effects of VC on the changes in $\mathrm{Ca}^{2+}$ concentration with Fluo-4/AM and Rhod-2/ $\mathrm{AM}$ as the cytosolic and mitochondrial $\mathrm{Ca}^{2+}$ indicators, respectively. Figure $6 \mathrm{~A}$ showed the effect of $\mathrm{VC}$ on cytosolic $\mathrm{Ca}^{2+}$ concentration. The fluorescence of the OGD/R group increased compared with the control group, but was significantly reduced in the VC group $(p<0.05)$. The decrease in cytosolic $\mathrm{Ca}^{2+}$ overload by VC was completely abolished by 5-HD or LND treatment. There were no obvious differences between the 5-HD, LND and OGD/R groups. As shown in Fig. 6B, post-treatment of VC significantly attenuated OGD/R-induced mitochondrial $\mathrm{Ca}^{2+}$ overload. This effect was abolished by the co-administration of 5-HD or LND $(p<0.05)$.

Effects of VC on OGD/R-Induced ROS Accumulation

The level of ROS in the OGD/R group significantly increased compared with the control group ( $p<0.05$; Fig. 6C). However, in cardiomyocytes, post-treatment with $\mathrm{VC}$ reduced the ROS levels $(p<0.05)$. The effect of VC was also inhibited by 5 -HD or LND (Fig. 6C).

Effects of VC on OGD/R-Induced mPTP Opening

To confirm the opening of $\mathrm{MPTP}$ and the inhibitory effect of $\mathrm{VC}$, we measured the fluorescence intensity in calcein/AMloaded cardiomyocytes. Compared with the control group, the $\mathrm{OGD} / \mathrm{R}$ group significantly reduced the fluorescence intensity. Post-treatment with $\mathrm{VC}$ for $3 \mathrm{~h}$ resulted in a significant increase in fluorescence intensity $(p<0.05)$, suggesting that $\mathrm{VC}$ alleviated mPTP opening in cardiomyocytes (Fig. 7A). Co-treatment with 5-HD or LND completely inhibited the VCinduced increase of fluorescence intensity. Compared with the OGD/R group, 5-HD or LND alone had no effect on mPTP opening.
Effects of VC on OGD/R-Induced $\Delta \Psi \mathrm{m}$ Dissipation

To determine whether the preservation of $\Delta \Psi \mathrm{m}$ was related to the protective effects of $\mathrm{VC}$, we evaluated the changes in TMRE fluorescence.

As shown in Fig. 7B, the OGD/R group significantly decreased TMRE fluorescence intensity compared with the control group, suggesting depolarization of $\Delta \Psi \mathrm{m}$. The decrease in TMRE fluorescence intensity was significantly suppressed by post-treatment with $\mathrm{VC}$, demonstrating the preservation of $\triangle \Psi \mathrm{m}$ (Fig. 7B). However, the VC-induced increase in TMRE fluorescence was completely abolished by exposure to 5-HD or LND. Taken together, these results indicated that VC prevented the depolarization of $\Delta \Psi \mathrm{m}$ in cardiomyocytes by activating mitoK $_{\text {ATP }}$ channels and inhibiting mPTP opening.

Effects of VC Involves PI3K-AKT Pathway Activation

VC significantly increased AKT $\left(\mathrm{Ser}^{473}\right)$ phosphorylation compared with the control group and OGD/R group $(p<0.05)$. The phosphorylation of AKT $\left(\mathrm{Ser}^{473}\right)$ was significantly decreased in the LY group compared with OGD/R group $(p<0.05)$. VC postconditioning-induced phosphorylation of Akt was suppressed by LY294002. VC+5-HD group had no difference with OGD/R group.

GSK3 $\beta\left(\operatorname{Ser}^{9}\right)$ phosphorylation was similar in control group, OGD/R group and LY group (Fig. $8 \mathrm{~B}$ ). VC post-conditioning improved the expression of $\mathrm{p}-\mathrm{GSK} 3 \beta\left(\mathrm{Ser}^{9}\right)$ compared with the OGD/R group $(p<0.05$, Fig. $8 \mathrm{~B})$. However, the $\mathrm{VC}+\mathrm{LY}$ group abolished the increase in $\mathrm{p}-\mathrm{GSK} 3 \beta$ ( $\left.\operatorname{Ser}^{9}\right)$ expression, which was induced by $\mathrm{VC}$. The phosphorylation of GSK $3 \beta$ was significantly elevated by VC post-conditioning compared to ischemic control and was completely abolished by LY294002. $\mathrm{VC}+5-\mathrm{HD}$ group had no significant difference with $\mathrm{OGD} / \mathrm{R}$ group.

Mitochondria are an important part of cellular ATP synthesis, and they provide energy for cardiomyocytes. Myocardial $\mathrm{I} / \mathrm{R}$ injury is caused when mitochondria are damaged and the mitochondrial permeability transition pore (mPTP) is open, which causes a loss in mitochondrial transmembrane point, respiratory chain rupture and apoptosis protein cytochrome $c$ release, eventually resulting in mitochondrial dysfunction and apoptosis. $^{20,21)}$ Reactive oxygen species and calcium overload can result in mPTP opening. Currently, many studies have 
A

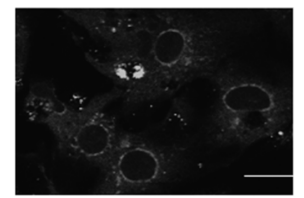

control

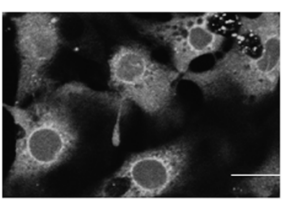

$\mathrm{VC}+5-\mathrm{HD}$

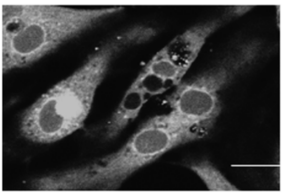

5-HD

B

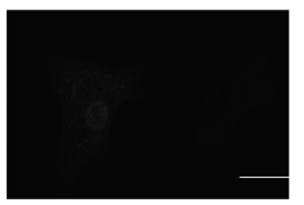

control

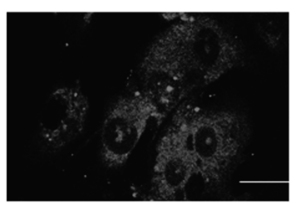

$\mathrm{VC}+5-\mathrm{HD}$

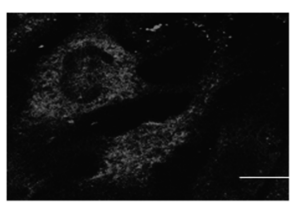

5-HD

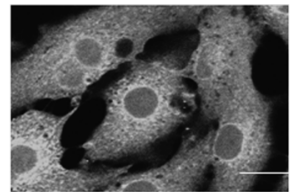

$\mathrm{OGD} / \mathrm{R}$

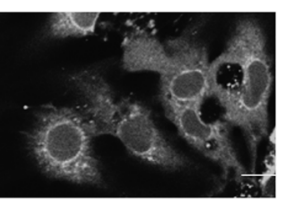

$V C+L N D$

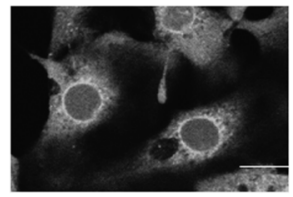

LND

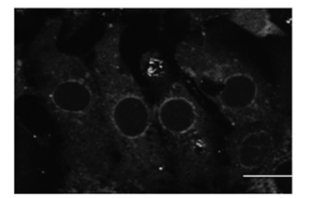

VC

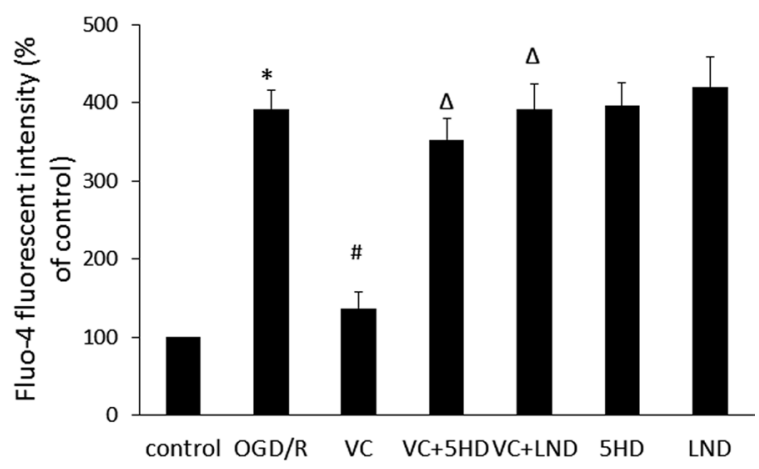

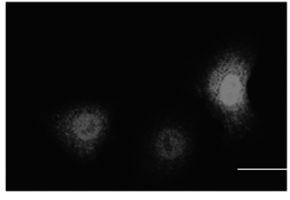

OGD/R

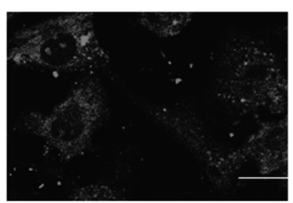

$V C+L N D$

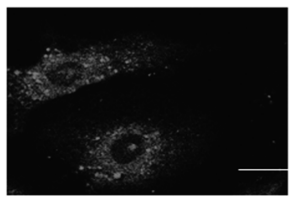

LND

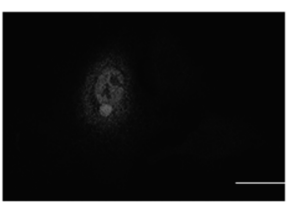

VC

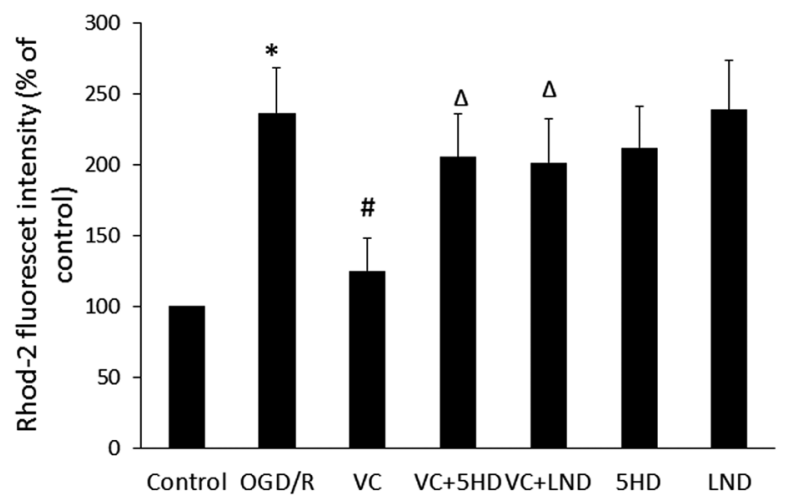

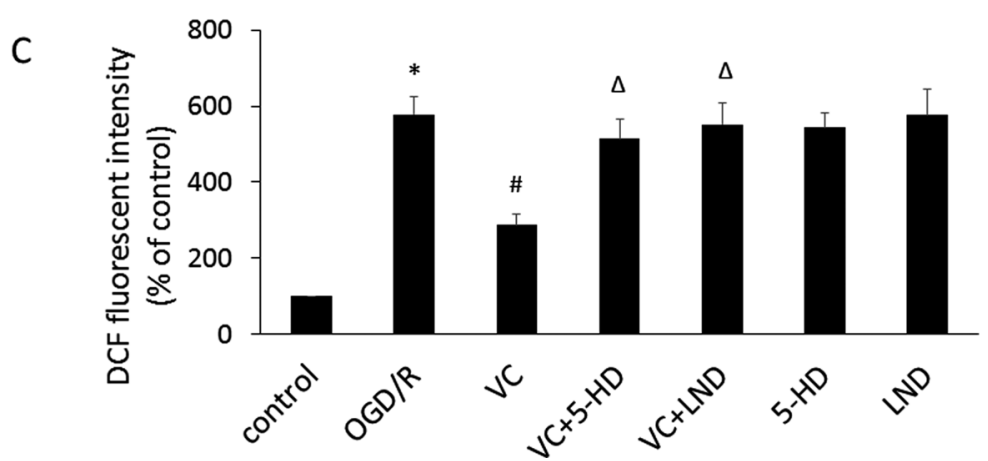

Fig. 6. Effects of VC on the Cytosolic $\mathrm{Ca}^{2+}$ Concentration (A), Mitochondrial $\mathrm{Ca}^{2+}$ Concentration (B) and Reactive Oxygen Species (ROS) Production (C) after OGD/R

Cells were treated as described in the protocol. Data are expressed as the mean \pm S.D., $n=5 .{ }^{*} p<0.05 v s$. control group; ${ }^{*} p<0.05 v s . \mathrm{I} / \mathrm{R}$ group; ${ }^{\Delta} p<0.05 v s$. VC group. I/R: ischemia/reperfusion; VC: vitamin C; 5-HD: 5-hydroxydecanoic acid; LND: lonidamine. Bar length, $25 \mu \mathrm{m}$. 


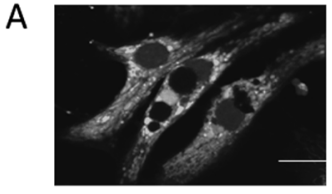

control

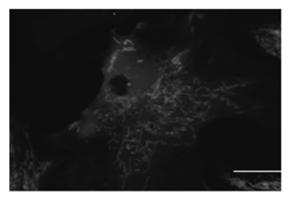

$\mathrm{VC}+5-\mathrm{HD}$

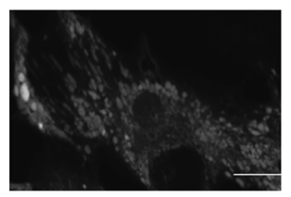

5-HD

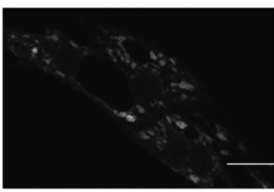

OGD/R

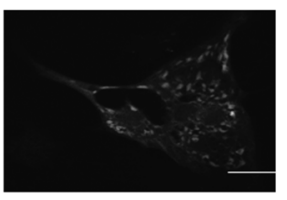

VC+LND

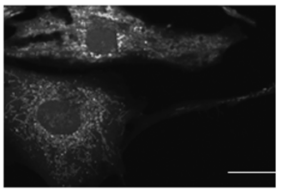

LND

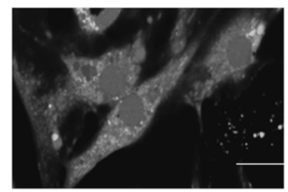

VC

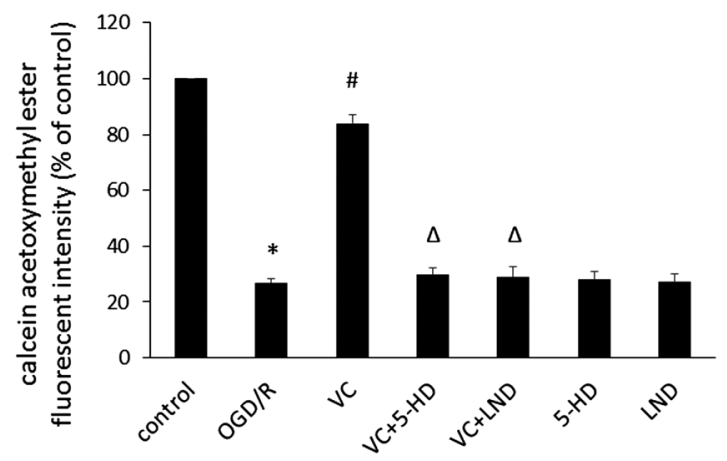

B

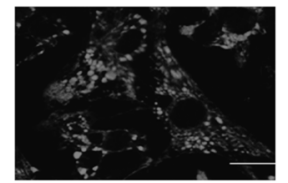

control

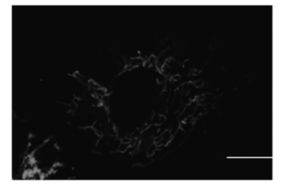

$\mathrm{VC}+5-\mathrm{HD}$

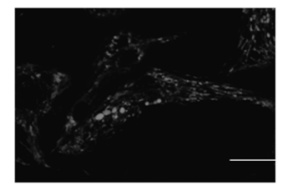

5-HD

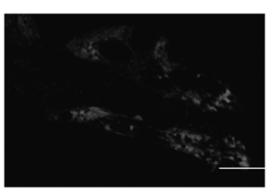

OGD/R

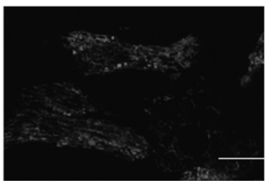

VC+LND

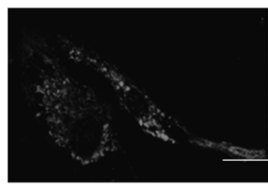

LND

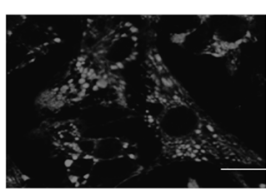

vc

Fig. 7. Effects of VC on the Mitochondrial Permeability Transition Pores (mPTP) Opening (A) and Mitochondrial Membrane Potential (B) after $\mathrm{OGD} / \mathrm{R}$

Cells were treated as described in the protocol. Data are expressed as the mean \pm S.D., $n=5 . * p<0.05 v s$. control group; ${ }^{*} p<0.05 v s$. I/R group; ${ }^{\Delta} p<0.05 v s$. VC group. I/R: ischemia/reperfusion; VC: vitamin C; 5-HD: 5-hydroxydecanoic acid; LND: lonidamine. Bar length, $25 \mu \mathrm{m}$.

confirmed that mitochondria are one of the important targets for heart protection. ${ }^{4,5)}$ There are two approaches to generate ROS in I/R: the xanthine oxidase pathway and mitochondrial respiratory chain approach. In myocardial ischemia hypoxia, a large number of hypoxanthine accumulates, and xanthine dehydrogenase converts into xanthine oxidase, in which the activity is low. After reperfusion, xanthine oxidase activity increases. Xanthine oxidase breaks down xanthine and generates many ROS. An increase in calcium overload can increase calcium-dependent protease activity, thereby promoting xanthine dehydrogenase conversion into xanthine oxidase and ROS. The mitochondrial respiratory chain is the major site of ROS production in the process of myocardial I/R. ${ }^{22)}$ The mitochondrial respiratory chain was uncoupled and $\mathrm{O}_{2}$ was de-acidized by a single electron and a large number of ROS was produced. ${ }^{23}$ This research showed that VC postprocessing can reduce ROS in myocardial $I / R$ and proved that
$\mathrm{VC}$ can remove free radicals, resulting in a protective effect on cardiomyocytes.

Calcium overload is one of the mechanisms of myocardial $\mathrm{I} / \mathrm{R}$ injury. Intracellular calcium overload in $\mathrm{I} / \mathrm{R}$ mainly contributes to abnormal $\mathrm{Na}^{+} / \mathrm{Ca}^{2+}$ exchange and biological membrane damage. A reduction in calcium overload can block or reduce myocardial injury, ${ }^{24)}$ which is consistent with our results. This study showed that VC post-treatment can significantly reduce the intracellular $\mathrm{Ca}^{2+}$ concentration. This alleviating effect of calcium overload was suppressed by the mitoK ${ }_{\mathrm{ATP}}$ channel blocker, 5-HD. We speculated that the effect of $\mathrm{VC}$ on reducing calcium overload is implemented via the opening of mitoK $\mathrm{ATP}_{\mathrm{P}}$ channels.

mPTP is a protein complex, which exists in the mitochondrial membrane and mainly consists of three components: voltage-dependent anion channel (VDAC) located in the outer membrane, cyclophilin D (CYPD) located in the matrix, and 
A

Con OGD/R VC VC +LY LY VC+5-HD 5-HD
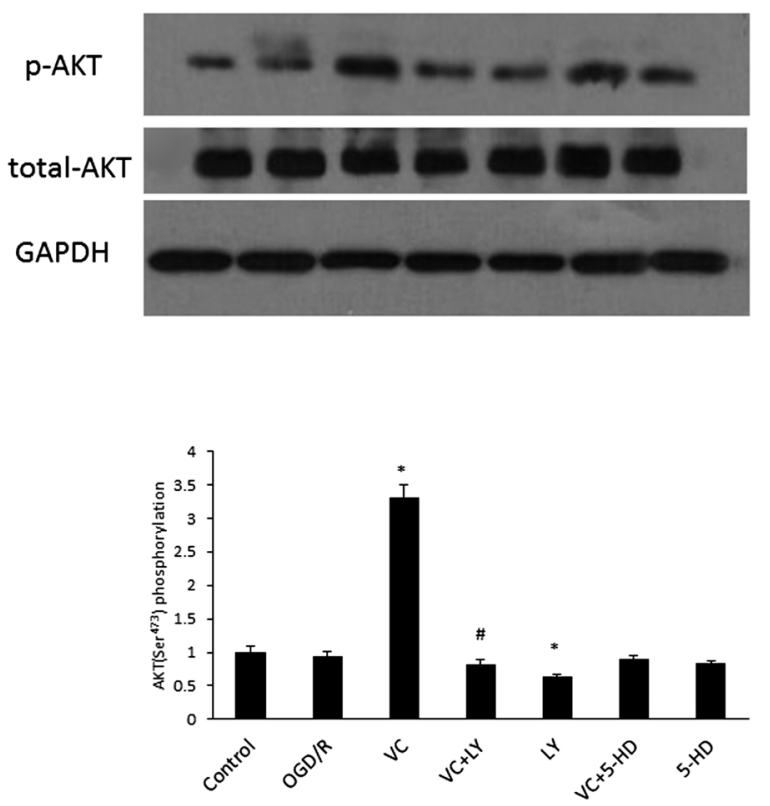

B Con OGD/R VC VC +LY LY VC +5-HD 5-HD

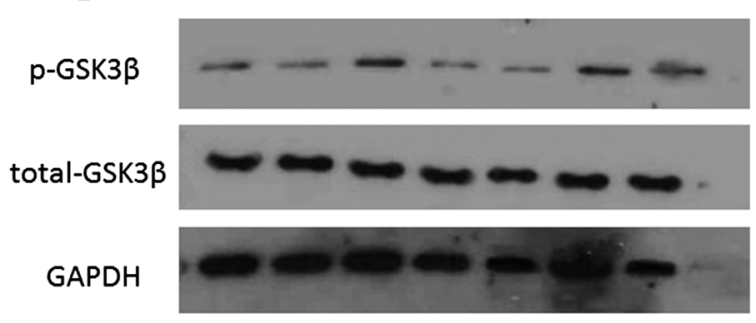

Fig. 8. Effects of VC Involves PI3K-AKT Pathway Activation

(A) Western blot for phosphor-AKT $\left(\mathrm{Ser}^{473}\right)$. (B) Western blot for phospho-GSK-3 $\beta\left(\mathrm{Ser}^{9}\right)$. Data are expressed as the mean \pm S.D., $n=5 .{ }^{*} p<0.05 v s . \mathrm{I} / \mathrm{R}$ group; ${ }^{*} p<0.05$ vs. VC group. I/R: ischemia/reperfusion; VC: vitamin C; 5-HD: 5-hydroxydecanoic acid; LND: lonidamine.

adenine nucleotide translocase (ANT) located in the inner membrane. In VDAC knockout mice and wild-type mice, Baines $e t a l{ }^{25)}$ reported no difference in the induction of ROS and calcium overload results in the opening of MPTP and the release of cytochrome $c$, suggesting that VDAC did not participate in the opening of mPTP. The combination of ANT and CYPD affect mPTP opening. CYPD knockout mice can antagonize cell apoptosis caused by reactive oxygen species and calcium overload-induced mPTP opening and alleviate myocardial I/R injury. ${ }^{26)}$

The maintenance of the $\Delta \Psi \mathrm{m}$ plays a pivotal role in the preservation of cellular homoeostasis and myocyte survival. A previous study showed that simvastatin post-processing can decrease the $\Delta \Psi \mathrm{m}$ depolarization, which was implemented via the opening of mitoK ${ }_{\text {ATP }}$ channels. ${ }^{3)}$ Membrane potential decline is mainly caused by mPTP opening. This study found that VC post-treatment can inhibit $\Delta \Psi \mathrm{m}$ decreases, and the effect was offset by the mitoK $\mathrm{K}_{\mathrm{ATP}}$ channel blocker 5-HD and $\mathrm{mPTP}$ opener LND. This finding proved that VC via the activation of mitoK $\mathrm{ATP}_{\mathrm{AT}}$ channels and opening of $\mathrm{MPTP}$ to reduce $\Delta \Psi \mathrm{m}$ depolarization, was consistent with the reported results.

The cardioprotective mechanism of VC post-treatment involves PI3K-Atk-GSK3 $\beta$ pathway activation, GSK3 $\beta$ ( $\operatorname{ser}^{9}$ ) phosphorylation and subsequent mitoK $\mathrm{K}_{\mathrm{ATP}}$ channels activation. Tsang et al. showed that inhibiting the activity of PI3K by given LY or wortmannin at reperfusion completely abolished post-treatment-induced myocardial protection, suggesting that post-treatment protects the myocardium by activating PI3KAkt. ${ }^{27)}$ Studies by Zhu et al. demonstrated that ischemic posttreatment increased the phosphorylation of Akt downstream targets GSK3 $\beta$ in remodeled hearts. ${ }^{19)}$ We demonstrated that VC post-treatment protects the myocardium against I/R injury by activating the kinase PI3K-Akt and its downstream targets GSK $3 \beta$. The activation of mitoK $\mathrm{ATP}_{\text {ATP }}$ channels has been demonstrated to mediate cardioprotective effects. We postulated that $\mathrm{VC}$ post-treatment protects the heart by activation of mitoK $_{\text {ATP }}$ channels via activation of Akt and GSK3 $\beta$. The results showed that $\mathrm{VC}$ myocardial protection disappeared when blocking the mitoK $_{\text {ATP }}$ channel, and the expression of pAkt $\left(\mathrm{Ser}^{473}\right)$ or pGSK-3 $\beta$ (Ser ${ }^{9}$ ) had a significant difference. That demonstrated that mitoK $_{\text {ATP }}$ channel located in the upstream of PI3K-AKT pathway.

\section{Conclusion}

The results of the current studies have shown that VC protects the myocardium from I/R-induced injury. This cardioprotective effect may be mediated by the activation of mitoK ${ }_{\text {ATP }}$ channels, a reduction in both $\mathrm{Ca}^{2+}$ overload and reactive oxygen species generation, the inhibition of $\mathrm{mPTP}$ opening and the maintenance of $\Delta \Psi \mathrm{m}$. Furthermore, mitoK $\mathrm{A}_{\mathrm{ATP}}$ channels may act upstream of mPTP. Vitamin $\mathrm{C}$ mediates cardioprotection via activation of the PI3K-Akt signaling pathway. This result may contribute towards the development of novel strategies for clinical cardioprotection against I/R injury.

Acknowledgment The work was supported by the Health Department of Hebei Province Key Science and Technology Research program (20120084).

Conflict of Interest The authors declare no conflict of interest.

\section{References}

1) Jennings R. B., Sommers H. M., Smyth G. A., Flack H. A., Linn H., Arch. Pathol., 70, 68-78 (1960).

2) Murry C. E., Jennings R. B., Reimer K. A., Circulation, 74, 1124$1136(1986)$

3) Shanmuganathan S., Hausenloy D. J., Duchen M. R., Yellon D. M., Am. J. Physiol. Heart Circ. Physiol., 289, H237-H242 (2005).

4) Fantinelli J. C., González Arbeláez L. F., Pérez Núñez I. A., Mosca 
S. M., Exp. Mol. Pathol., 94, 277-284 (2013).

5) Penna C., Perrelli M. G., Pagliaro P., Antioxid. Redox Signal., 18, 556-599 (2013)

6) Fantinelli J. C., Perez N. I. A., Gonzalez A. L. F., Schinella G. R., Mosca S. M., Int. J. Cardiol., 166, 173-180 (2013).

7) Louch W. E., Sheehan K. A., Wolska B. M., J. Mol. Cell. Cardiol., 51, 288-298 (2011).

8) Roth S., Dreixler J. C., Shaikh A. R., Lee K. H., Bindokas V., Invest. Ophthalmol. Vis. Sci., 47, 2114-2124 (2006).

9) Liu Y., Circulation, 99, 3043 (1999).

10) Griffiths E. J., Halestrap A. P., Biochem. J., 307, 93-98 (1995)

11) Perrelli M. G., Pagliaro P., Penna C. World, J. Cardiol., 3, 186-200 (2011).

12) Salie R., Moolman J. A., Lochner A., Basic Res. Cardiol., 107, 281 (2012).

13) Rajesh K. G., Sasaguri S., Zhitian Z., Suzuki R., Asakai R., Maeda H., Cardiovasc. Res., 59, 297-307 (2003).

14) Takeda T., Akao M., Matsumoto-Ida M., Kato M., Takenaka H., Kihara Y., Kume T., Akaike A., Kita T., J. Am. Coll. Cardiol., 47, 1882-1890 (2006).

15) Viswanath K., Bodiga S., Balogun V., Zhang A., Bodiga V. L., Biometals, 24, 171-180 (2011).

16) Wu Q., Li H., Wu Y., Shen W., Zeng L., Cheng H., He L., J. Ethnopharmacol., 138, 34-39 (2011).
17) Domoki F., Kis B., Gaspar T., Snipes J. A., Bari F., Busija D. W., Neurochem. Int., 56, 404-409 (2010).

18) Zhao Z. Y., Luan P., Huang S. X., Xiao S. H., Zhao J., Zhang B. Gu B. B., Pi R. B., Liu J., CNS Neurosci. Ther., 19, 163-169 (2013).

19) Zhu J., Rebecchi M. J., Glass P. S., Brink P. R., Liu L., Am. J. Physiol. Heart Circ. Physiol., 300, H922-H930 (2011).

20) Gustafsson A. B., Gottlieb R. A., Cardiovasc. Res., 77, 334-343 (2008).

21) Hausenloy D., Wynne A., Duchen M., Yellon D., Circulation, 109, 1714-1717 (2004).

22) Vanden Hoek T. L., Becker L. B., Shao Z., Li C., Schumacker P. T., J. Biol. Chem., 273, 18092-18098 (1998).

23) Kevin L. G., Novalija E., Stowe D. F., Anesth. Analg., 101, 12751287 (2005)

24) Ngoh G. A., Watson L. J., Facundo H. T., Jones S. P., Amino Acids, 40, 895-911 (2011).

25) Baines C. P., Kaiser R. A., Sheiko T., Craigen W. J., Molkentin J. D., Nat. Cell Biol., 9, 550-555 (2007).

26) Nakagawa T., Shimizu S., Watanabe T., Yamaguchi O., Otsu K. Yamagata H., Inohara H., Kubo T., Tsujimoto Y., Nature (London), 434, 652-658 (2005).

27) Tsang A., Hausenloy D. J., Mocanu M. M., Yellon D. M., Circ. Res., 95, 230-232 (2004). 